\title{
Le chimiotactisme des spermatozoïdes a-t-il un rôle dans la fécondation?
}

\author{
M.EISENBACH \\ Department of Biological Chemistry, The Weizmann Institute of Science, Israel
}

\section{RÉSUMÉ}

Le chimiotactisme des spermatozoïdes par le fluide folliculaire a été établi par différents moyens en ce qui concerne les spermatozoïdes de l'homme et de la souris. Seule une petite fraction d'une population donnée de spermatozoïdes (en moyenne environ 10\%) présente une sensibilité chimiotactique, et cette fraction constitue les spermatozoïdes capacités. La sensibilité chimiotactique et l'état capacité sont tous les deux transitoires (avec une durée de vie de 50 minutes à 4 heures) et ne surviennent qu'une seule fois dans la vie d'un spermatozoïde. On a proposé comme rôle au chimiotactisme des spermatozoïdes chez les mammifères (du moins chez l'homme) le recrutement sélectif de spermatozoïdes capacités pour la fécondation de l'ovocyte ; le rôle du remplacement continu des spermatozoïdes sensibles au chimiotactisme / capacités serait de prolonger la durée de temps pendant laquelle des spermatozoïdes capacités seraient disponibles dans le tractus génital féminin. Les substances chimio-attractives des spermatozoïdes n'ont pas été identifiées mais elles semblent être des peptides thermo-stables. La localisation in vivo du chimiotactisme des spermatozoïdes est inconnue ; de nombreuses localisations potentielles sont discutées dans cet article.

Mots clés : chimiotactisme ; capacitation ; chimiokinésie ; fécondation ; mobilité des spermatozoïdes ; appareil reproducteur féminin.

\section{INTRODUCTION}

La question ici posée est de savoir si, dans l'espèce humaine, l'interaction spermatozoïde ovule est la conséquence d'une collision au hasard ou bien si elle est le résultat d'une communication préalable entre eux, plus spécifique - le chimiotactisme. Le chimiotactisme est le processus par lequel la direction du mouvement des cellules est majorée par le gradient d'une substance chimio-attractive ou minorée par le gradient d'une substance chimio-répulsive. Cet article résume les études publiées sur le chimiotactisme des spermatozoïdes chez l'humain. Il démontre la survenue du chimiotactisme des spermatozoïdes et conclut que l'interaction spermatozoïde - ovule n'est pas une coïncidence.

\section{LE CHIMIOTACTISME DES SPERMA- TOZOÏDES DE MAMMIFÈRES EST-IL UNE NÉCESSITÉ ?}

La plupart des études sur le chimiotactisme des spermatozoïdes ont été réalisées sur des espèces marines, essentiellement chez l'oursin de mer. Dans ce cas, la nécessité du chimiotactisme est évidente : les femelles pondent leurs œufs dans l'eau de mer avant la fécondation, et les spermatozoïdes doivent les trouver. Les spermatozoïdes atteignent les ovocytes par

Correspondance : Michael Eisenbach Department of Biological Chemistry, The Weizmann Institute of Science, 76100 Rehovot, Israel.

E-mail : M.EISENBACH@WEIZMANN.AC.IL 
guidage chimiotactique, à savoir en nageant dans le courant d'une substance chimio-attractive libérée à partir de l'ovocyte [1-3]. Cependant, chez les mammifères, la fécondation est interne et un nombre important de spermatozoïdes est éjaculé directement dans l'appareil reproducteur femelle : 4 à 40 x 107 dans l'espèce humaine [4]. On croyait donc que le chimiotactisme n'était pas nécessaire parce qu'un nombre suffisant de spermatozoïdes pouvait atteindre l'ovule par pure coïncidence. Cette conclusion est-elle correcte?

A l'opposée de cette conception ancienne, le nombre de spermatozoïdes qui atteignent réellement la trompe est extrêmement faible, d'un ordre de grandeur qui est six fois moindre que le nombre de spermatozoïdes déposés dans le vagin [5-7]. Chez l'humain, par exemple, seuls environ 250 (fourchette : 80-1400) spermatozoïdes sont retrouvés dans les deux trompes [7]. Ce faible nombre réduit à des valeurs irréelles la chance d'une collision par pure coïncidence entre les spermatozoïdes et l'ovule dans l'ampoule tubaire. De plus, alors que le nombre de spermatozoïdes retrouvés n'est pas significativement différent entre la trompe ovulatoire et la trompe non ovulatoire, il existe une distribution significativement différente des spermatozoïdes à l'intérieur des trompes : l'ampoule tubaire ovulatoire, où se produit la fécondation, contient un nombre de spermatozoïdes significativement plus important que l'ampoule tubaire controlatérale [7]. Ces résultats défient encore une fois l'explication fournie par l'ancien dogme. Le chimiotactisme peut être l'un des mécanismes responsables de cette distribution des spermatozoïdes.

Par ailleurs, chez la souris, le rat et le porc, la distribution initiale des spermatozoïdes au niveau du site de fécondation tend à être approximativement de un spermatozoïde par ovocyte pour la plupart des ovocytes de la masse cellulaire du cumulus [ 8 et autres références de cet article]. Cependant, le nombre de spermatozoïdes peut augmenter lentement et progressivement avec le temps écoulé depuis lovulation Le fait que de multiples spermatozoïdes n'arrivent pas ensemble vers un unique ovocyte, et qu'aucun ovocyte ne soit dépourvu de spermatozoïdes, suggère que chaque sper- matozoïde est guidé vers un unique ovocyte et qu'il existe un mécanisme (peut être de chimiotactisme négatif) indiquant qu'un ovocyte vient juste d'être activé par un spermatozoïde et empêchant l'arrivée d'autres spermatozoïdes (au moins pour une courte période).

A partir de ces données de base, qui suggèrent indirectement la nécessité d'un chimiotactisme des spermatozoïdes, plusieurs laboratoires ont étudié le chimiotactisme des spermatozoïdes chez les mammifères.

\section{COMMENT MESURE-T-ON LE CHIMIOTACTISME DES SPERMATOZOÏDES ?}

Comme il est très difficile, sinon impossible, de réaliser des mesures directes in vivo du chimiotactisme des spermatozoïdes de mammifères, toutes les études publiées ont été menées in vitro. La plupart des études sur le chimiotactisme des spermatozoïdes impliquent l'accumulation des spermatozoïdes dans le fluide folliculaire, essentiellement parce que le fluide folliculaire contient des sécrétions qui proviennent de l'ovocyte et des cellules environnantes, mais aussi en raison de son accessibilité. On doit cependant remarquer que le fluide per se n'a probablement pas de rôle physiologique après l'ovulation vu les faibles quantités transportées dans la trompe [9].

La littérature concernant le chimiotactisme des spermatozoïdes par le fluide folliculaire apparaît quelque peu confuse en raison de l'état contradictoire des résultats rapportés. L'une des raisons principales de cette situation est que certaines études mesurent l'accumulation des spermatozoïdes sans réaliser que ce n'est pas seulement le chimiotactisme qui peut entraîner l'accumulation. La chimiokinésie (augmentation de la vitesse) et le piégeage (soit piégeage physiologique comme par exemple un effet négatif du fluide folliculaire sur la mobilité, soit piégeage mécanique comme l'adsorption à la chambre de mesure) peuvent aussi être des causes d'accumulation des spermatozoïdes. Les techniques qui ont été utilisées pour mesurer le chimiotactisme des spermatozoïdes in vitro (Figure 1) peuvent être réparties dans quatre catégories et seules deux 

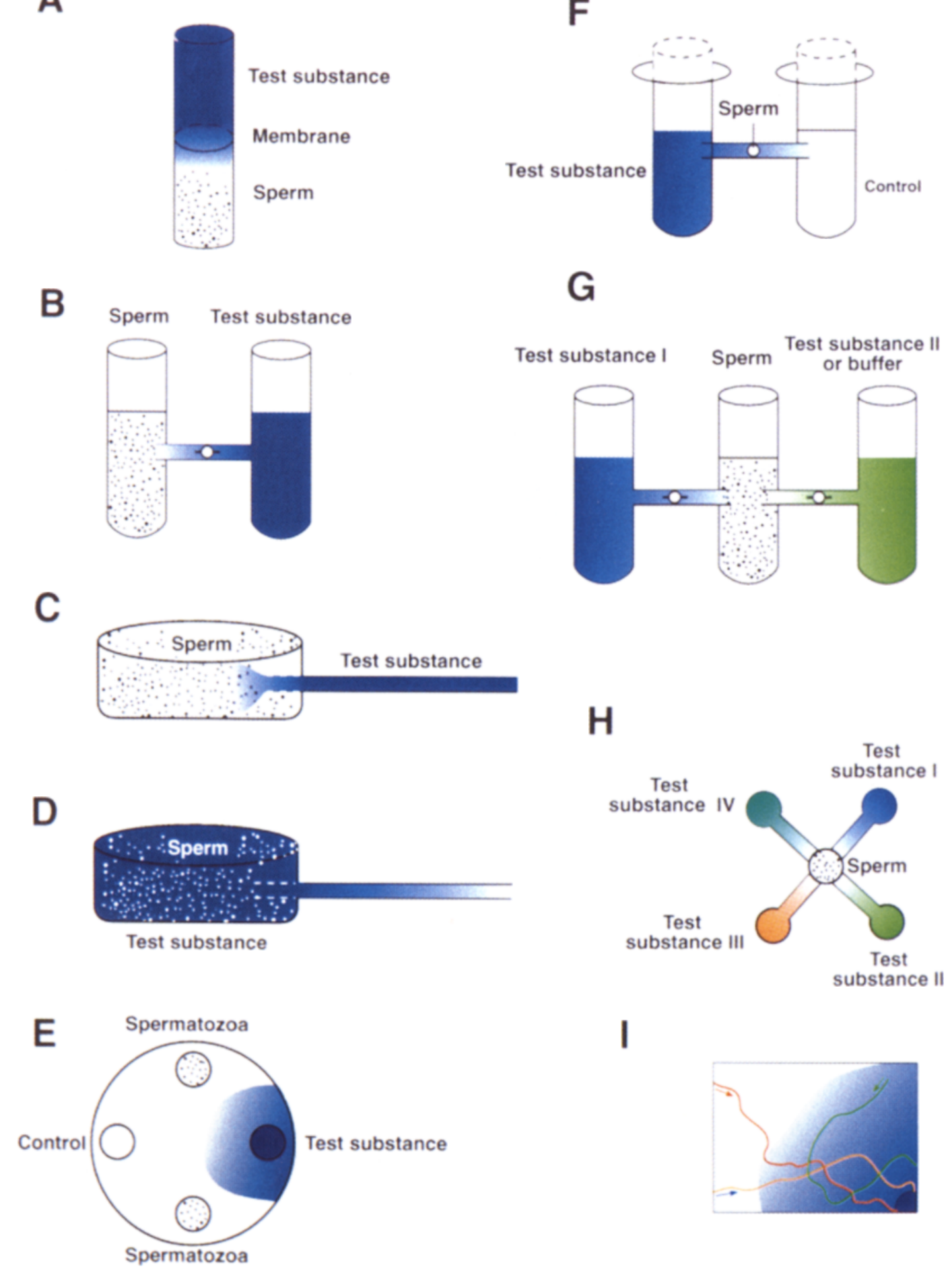

Test substance

substance II

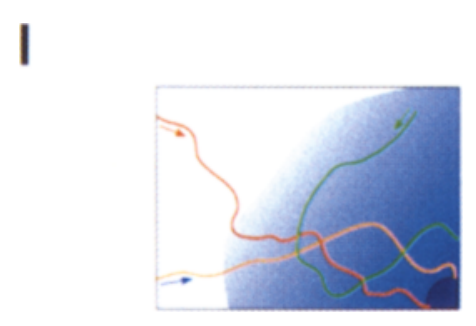

Figure 1 : Différentes techniques et protocoles utilisés pour étudier l'accumulation et le chimiotactisme. A. Une technique d'accumulation dans un appareil constitué de deux puits séparés par une fine membrane de polycarbonate [33]. B. Une technique d'accumulation dans un appareil constitué de deux puits connectés par un tube [18]. C. Accumulation des spermatozoüdes par une technique capillaire [11]. D. Une technique capillaire inversée [11]. E. Une technique "du choix" microscopique dans une chambre scellée (vue par en dessus) [12]. F. Une technique "du choix" dans un appareil constitué de deux puits [38]. G. Une technique " du choix" dans un appareil constitué de trois puits [16]. H. Vue par en dessus d'un appareil constitué de cinq puits pour une technique "du choix "[39,42]. I. Parcours schématique de spermatozoïdes humains dans un gradient attractif [11] (avec la bienveillante autorisation de [3]). 
d'entre elles peuvent faire la différence entre le chimiotactisme et les deux autres processus (Tableau 1).

La première catégorie des techniques de mesure du chimiotactisme est l'accumulation des spermatozoïdes dans un gradient ascendant de chimoattracteur (Figure 1a-c). Dans cette cątégorie de techniques macroscopiques, les spermatozoïdes détectent un gradient croissant de chimioattracteur et vont s'accumuler à proximité ou au niveau même de la source. Le gradient chimioattracteur est établi par diffusion. De nombreuses variantes de ce type de technique ont été publiées $[3,10]$, utilisant différents dispositifs, par exemple un capillaire rempli du chimioattracteur et immergé dans un puits contenant la suspension de spermatozoïdes [11]. Le principal inconvénient de ce type de techniques est qu'elles ne peuvent pas différencier le chimiotactisme des autres causes d'accumulation des spermatozoïdes (Tableau 1).

La deuxième catégorie de techniques implique une technique opposée, à savoir l'accumulation dans un gradient décroissant de chimioattracteur (Figure 1d). Dans cette configuration, les spermatozoïdes placés dans un puits sont mis en suspension dans une solution qui contient déjà le chimioattracteur, avec un capillaire connecté au puits qui contient soit un tampon témoin soit le chimioattracteur. Quand le capillaire contient un tampon, les spermatozoïdes détectent un gradient décroissant du chimioattracteur lorsqu'ils se déplacent du puits au capillaire. Quand le chimioattracteur est à la fois dans le capillaire et dans le puits, ils ne détectent aucun gradient. Cette technique mesure ainsi la tendance des spermatozoïdes à quitter le chimioattracteur plutôt qu'à s'accumuler dans le chimioattracteur. Ce type de technique fait la différence entre chimiotactisme, chimiokinésie et piégeage (Tableau 1), puisque seul le chimiotactisme (à la différence des effets de la chimiokinésie et du piégeage) est dépendant de la présence d'un gradient chimique.

La troisième catégorie comprend la technique " du choix". C'est l'une des techniques les plus employées, dans laquelle les spermatozoïdes choisissent entre deux puits (ou deux chambres), l'un contenant le chimioattracteur et l'autre contenant un tampon servant de témoin. De nombreuses variantes de cette sorte ont été publiées : une chambre scellée pour la mesure microscopique (Figure 1e), et pour la réalisation de mesures macroscopiques - des dispositifs avec 2, 3 ou 5 puits ou chambres, connectés avec un tube (Figure 1fh). De telles techniques peuvent différencier le chimiotactisme de la chimiokinésie, mais elles

Tableau 1. Catégories de techniques utilisées pour mettre en évidence le chimiotactisme des spermatozoïdes humains $a$

\section{Catégories}

\section{Distinction entre chimiotactisme et chimiokinésie}

\section{Distinction entre chimiotactisme et piégeage \\ Références}
A. Accumulation des spermatozoïdes

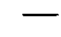
- gradient croissant
B. Accumulation des spermatozoïdes
— gradient décroissant
C. Techniques “ du choix"
C. Analyse des trajectoires
des spermatozoïdes
$[11,18,32-36]$
$[11,37]$
$[11-13,15,16,18,38-44]$
$[11,16,17,19,45]$

$a$ Avec la permission de [46]. 
ne peuvent pas différencier le chimiotactisme du piégeage (Tableau 1 ).

La quatrième catégorie de techniques consiste à suivre les trajectoires parcourues par les spermatozoïdes. Le chimiotactisme, à la différence de la chimiokinésie et du piégeage, implique des changements directionnels typiques du mouvement des spermatozoïdes en direction de la source de chimioattracteur. C'est pourquoi une technique essentielle pour différencier le chimiotactisme des autres processus d'accumulation des spermatozoïdes est d'étudier les trajectoires faites par les spermatozoïdes dans un gradient de chimioattracteur (Figure 1i).

Comme cela est montré dans le Tableau 1, seules les catégories $B$ et $D$ font la différence entre chimiotactisme et les autres processus qui peuvent être la cause d'une accumulation des spermatozoïdes. De façon regrettable, la plupart des laboratoires n'utilisent pas ces techniques.

\section{FAITS CONCERNANT LA SURVENUE CHEZ LES MAMMIFÈRES DU CHIMIO- TACTISME DES SPERMATOZOÏDES}

Les résultats des études de chimiotactisme par le fluide folliculaire sont résumés dans le Tableau 2. Etant données les mises en garde précédentes, toutes les études ne prouvent pas actuellement la survenue d'un chimiotactisme des spermatozoïdes par le fluide folliculaire. Le Tableau indique pour chaque étude si une distinction a été faite entre le chimiotactisme et d'autres processus responsables de l'accumulation des spermatozoïdes. Selon que cette distinction a été faite ou non, les résultats de toutes les études, à l'exception d'une seule, sont cohérents et démontrent la survenue d'un chimiotactisme des spermatozoïdes par le fluide folliculaire (Tableau 2). Les raisons des résultats négatifs de Makler et al. [12,13] essentiellement une dilution insuffisante du fluide folliculaire - sont discutées dans un article cité [10]. Ainsi, le chimiotactisme des spermatozoïdes in vitro par le fluide folliculaire est un fait au moins chez l'homme et la souris.

\section{CHIMIOATTRACTEURS DU FLUIDE FOLLICULAIRE}

L'identité des chimioattracteurs contenus dans le fluide folliculaire n'est pas connue. On a identifié [11] une fraction active du fluide folliculaire qui contient les chimioattracteurs (certainement des peptides thermiquement stables [14]). Beaucoup d'autres chimioattracteurs du fluide folliculaire ont été rapportés dans la littérature, mais aucun n'a été confirmé (voir référence bibliographique [3] pour une revue). Par exemple, on a récemment démontré que l'accumulation des spermatozoïdes dans la progestérone, rapportée par Villanueva- Diaz et al. [15] comme le chimioattracteur majeur du fluide folliculaire, résultait principalement du piégeage en raison d'une mobilité de type hyper activée et non pas du chimiotactisme [16].

On doit remarquer que le fluide folliculaire contient un certain nombre de substances qui sont sécrétées dans le follicule avant l'ovulation, alors que le chimiotactisme des spermatozoïdes survient probablement après l'ovulation. C'est pourquoi les stimuli chimiotactiques sécrétés à partir de l'ovocyte ou du cumulus oophore après l'ovulation doivent être différents de ceux du fluide folliculaire. En effet, on a démontré chez la souris que le fluide de la trompe, comme le fluide folliculaire, attirait les spermatozoïdes par chimiotactisme, ce qui soulève la possibilité de la survenue d'un chimiotactisme séquentiel sur le parcours des spermatozoïdes vers l'ovocyte [17].

\section{CARACTÉRISTIQUES DU CHIMIO- TACTISME DES SPERMATOZOÏDES.}

Le chimiotactisme des spermatozoïdes de mammifêres a de nombreuses caractéristiques uniques :

a) la fraction des spermatozoïdes chimiotactiques dans une population donnée de spermatozoïdes est faible (2-12\% chez l'humain [18], et environ $10 \%$ chez la souris $[17,19]$ ) ;

b) la sensibilité des spermatozoïdes au chimiotactisme est temporaire et n'est acquise qu'une seule fois dans la vie d'un spermatozoïde [18] ; 
Tableau 2 : Chimiotactisme des spermatozoüdes de mammiferes par le fluide folliculaire a

Etudes b

Espèces

$\begin{array}{ll}\text { Observation } & \begin{array}{l}\text { Catégorie } \\ \text { de }\end{array} \\ \text { d'un } & \text { technique c } \\ \text { chimiotactisme } & \text { technarent }\end{array}$

Distinction entre chimiotactisme et tous les autres processus responsables de l'accumulation
Villanueva-Díaz et al. [42]

\section{Humain}

Humain

Humain

Makler et al. [12, 13]

Villanueva-Díaz et al. [38]

Ralt et al. [11]

Cohen-Dayag et al. [18]

Navarro et al. [47]

Giojalas and Rovasio [19]

Oliveira et al. [17]

Jaiswal et al. [16]
Humain

Humain

Humain

Cheval

Souris

Souris

Humain
$+$

$+$

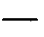

$+$

$+$

$+$

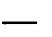

$+$

$+$

$+$
C

A

C

A

A B C D $\quad+$

A

C

D

D

C D

a Avec la permission de [46].

b Ordre chronologique. Les études antérieures non concluantes [revues par Eisenbach and Tur-Kaspa [10]] n'ont pas été incluses dans le tableau.

c En fonction du Tableau 1. 
c) il y a un remplacement continu des spermatozoïdes chimiotactiques au sein d'une population de spermatozoïdes - les spermatozoïdes chimiotactiques perdent leur activité alors que d'autres l'acquièrent [18]. Ces observations soulevèrent la possibilité que les spermatozoïdes ne soient sélectivement chimiotactiques qu'à une certaine étape physiologique, probablement au stade de la capacitation [20]. Ceci signifie que, au moins chez l'humain, des spermatozoïdes acquièrent leur sensibilité chimiotactique au cours du processus de capacitation et perdent cette sensibilité quand l'état capacité est terminé [20]. L'exactitude de cette possibilité a été prouvée en utilisant le chimiotactisme pour séparer une sous - population de spermatozoïdes enrichie en spermatozoïdes sensibles au chimiotactisme d'une sous-population non enrichie, et en établissant une corrélation étroite et synchrone entre les taux de spermatozoïdes capacités et ceux sensibles au chimiotactisme [21]. L'obtention de ces souspopulations enrichie et non enrichie a conduit à la détermination que l'état capacité des spermatozoïdes humains n'était pas statique mais plutôt transitoire avec une durée de vie de 50 à 240 minutes in vitro, et qu'il existait un processus continu de remplacement des spermatozoïdes capacités au sein d'une population de spermatozoïdes [21]. La conséquence de ce remplacement continu des spermatozoïdes capacités est l'existence d'une population hétérogène de spermatozoïdes, qui comprend des cellules qui n'ont pas encore débuté le processus de capacitation, des cellules à diverses étapes de la capacitation, des cellules pleinement capacitées, des cellules post-capacitées et des cellules ayant fait leur réaction acrosomique [22,23]. L'association entre sensibilité au chimiotactisme et état capacité repose sur

a) la similarité des pourcentages de spermatozoïdes chimiotactiques et capacités dans une population donnée de spermatozoïdes,

b) la similarité de la cinétique de remplacement des spermatozoïdes capacités et chimiotactiques, et

c) le fait qu'une déplétion délibérée en spermatozoïdes capacités entraîne une perte totale du chimiotactisme et, vice versa, une déplétion en spermatozoïdes chimiotactiques entraîne une déplétion des spermatozoïdes capacités [21]. La découverte récente chez la souris, comme chez l'humain, qu'environ seuls $10 \%$ des spermatozoides étaient sensibles au chimiotactisme [17-19], suggèrerait que l'association entre sensibilité au chimiotactisme et état capacité a lieu aussi chez la souris.

\section{ROLE PHYSIOLOGIQUE DU CHIMIOTACTISME DES SPERMATOZOÏDES.}

Le rôle que joue le chimiotactisme des spermatozoïdes in vivo est difficile à préciser étant donné l'absence de données in vivo. L'association de la sensibilité au chimiotactisme avec l'état capacité a soulevé la possibilité que, in vivo, le rôle du chimiotactisme des spermatozoïdes humains n'était pas de diriger un grand nombre de spermatozoïdes vers l'ovocyte, mais plutôt de recruter une population sélectionnée de spermatozoïdes, c'est à dire des spermatozoïdes capacités, pour féconder l'ovocyte. Un rôle éventuel du remplacement continu des spermatozoïdes capacités pourrait être d'assurer la disponibilité en spermatozoïdes capacités sur une longue période de temps, étant donné la faible durée de l'état capacité de chaque spermatozoïde [20,21].

\section{LOCALISATIONS POTENTIELLES DU CHIMIOTACTISME DES SPERMATO- ZOÏDES IN VIVO.}

Le site où a lieu le chimiotactisme des spermatozoïdes in vivo est inconnu. Un petit nombre de possibilités potentielles semble raisonnable :

\section{Entre l'isthme et l'ampoule.}

$\mathrm{Au}$ moins chez les mammifères non humains, une fraction considérable des spermatozoïdes éjaculés dans l'appareil reproducteur femelle est retenue avec une mobilité réduite dans des sites de stockage (habituellement l'isthme de la trompe). Apparemment, quand les spermatozoïdes remontent l'isthme tubaire, ils rencontrent une lumière étroite contenant une grande quantité de mucus qui gêne leur progression. Les spermatozoïdes viennent fréquemment au contact de l'épithélium tubaire où ils peuvent se lier fortement aux structures carbohydratées portées par des glycoprotéines 
ou des glycolipides à la surface de l'épithélium tubaire et par conséquent y être stockés [24]. Quand survient l'ovulation, certains des spermatozoïdes localisés dans le réservoir à spermatozoïdes retrouvent une mobilité élevée et parcourent la distance entre le site de stockage et le site de fécondation au niveau de l'ampoule tubaire en quelques minutes $[6,8,25]$. Seuls les spermatozoïdes capacités se détachent de l'épithélium et sont libérés du site de stockage $[26,27]$. Une possibilité serait l'implication du chimiotactisme dans l'orientation en direction de l'ovocyte des spermatozoïdes capacités libérés. Ceci pourrait être important vu le faible nombre de spermatozoïdes libérés du site de stockage [8]. Cependant, en raison des contractions tubaires discutées ci-dessus, un gradient de chimioattracteur ne peut probablement pas être établi sur de grandes distances dans la trompe, c'est à dire que l'étendue du chimiotactisme dans la trompe doit être relativement courte. C'est pourquoi, si le chimiotactisme survient entre le site de stockage de l'isthme et l'ampoule tubaire, il doit être constitué de plusieurs étapes, chaque étape dirigeant séquentiellement les spermatozoïdes capacités sur une étendue relativement courte (comme cela a été récemment suggéré pour le chimiotactisme des spermatozoïdes chez la souris [17]).

\section{Dans l'ampoule, au voisinage du com- plexe cumulus = ovocyte.}

$\mathrm{Au}$ vu des observations suggérant que le chimiotactisme des spermatozoïdes dans la trompe puisse avoir une faible étendue, il est possible que le chimiotactisme ne survienne que tout près du complexe cumulus - ovocyte. Une observation ancienne, le cumulus oophorus sécrète une substance qui altère le profil $\mathrm{du}$ mouvement spermatique [28], est cohérente avec cette possibilité.

\section{A l'intérieur du cumulus.}

Le cumulus oophorus, qui entoure l'ovocyte, fournit un milieu visco-élastique qui résiste aux mouvements de la trompe [18]. Les découvertes

a) que chez les mammifêres, les quelques premiers spermatozoïdes qui entrent dans le cumulus trouvent d'une façon très efficace l'ovocyte [29], b) que seuls les spermatozoïdes capacités peuvent pénétrer le cumulus oophorus (pour revue, voir [30] et [31]), et

c) que la distribution initiale de spermatozoïdes au niveau du site de fécondation tend habituellement à être approximativement de un spermatozoïde par ovocyte parmi la plupart des ovocytes de la masse cellulaire du cumulus [8] - sont en accord avec la possibilité d'un chimiotactisme des spermatozoïdes à l'intérieur du cumulus.

\section{IMPLICATIONS CLINIQUES.}

Etant donné la notion que le chimiotactisme est nécessaire à la sélection des spermatozoïdes capacités et compte tenu de la découverte que seuls les fluides folliculaires des follicules dont les ovocytes peuvent être fécondés sont chimiotactiquement actifs [32], une communication préalable défaillante entre les spermatozoïdes et l'ovocyte peut être une cause d'infertilité. Il est raisonnable de penser que, dans le futur, le chimiotactisme puisse être exploité comme un outil diagnostique de la qualité des spermatozoïdes et de l'infécondité masculine, et puisse être utilisé comme une procédure biologique de sélection des spermatozoïdes avant fécondation in vitro (FIV). En particulier, il pourrait être utilisé avant FIV avec micro - manipulation (injection intra-cytoplasmique d'un unique spermatozoïde (ICSI)) ou lors d'insémination intra-utérine. Tout aussi excitante est la possibilité que l'empêchement du chimiotactisme des spermatozoïdes puisse devenir une nouvelle approche en contraception.

\section{REFERENCES}

1. MILLER, R.L. (1985). Sperm chemo-orientation in the metazoa. In: Biology of Fertilization (C.B. Metz and A. Monroy, eds.), pp. 275-337. Academic Press, New York.

2. COSSON, M.P. (1990). Sperm chemotaxis. In: Controls of Sperm Motility: Biological and Clinical Aspects (C. Gagnon, ed.) pp. 103-135. CRC Press, Boca Raton, FL.

3. EISENBACH, M. (1999). Sperm chemotaxis. Rev. Reprod. 4, 56-66.

4. World Health Organization (1993). "Laboratory manual for the examination of human semen and semen-cervical mucus interaction." Cambridge University Press, New York. 
5. HARPER, M.J.K. (1982). Sperm and egg transport. In: Germ Cells and Fertilization (C.R. Austin and R.V. Short, eds.), pp. 102-127. Cambridge University Press, Cambridge, England.

6. BARRATT, C.L.R. AND COOKE, I.D. (1991). Sperm transport in the human female reproductive tract - a dynamic interaction. Int. J. Androl. 14, 394-411.

7. WILliams, M., HILL, C.J., SCUDAMORE, I., DUNPHY, B., COOKE, I.D. AND BARRATT, C.L.R. (1993). Sperm numbers and distribution within the human Fallopian tube around ovulation. Hum. Reprod. 8, 2019-2026.

8. HUNTER, R.H.F. (1993). Sperm:egg ratios and putative molecular signals to modulate gamete interactions in polytocous mammals. Mol. Reprod. Dev. 35, 324-327.

9. HANSEN, C., SRIKANDAKUMAR, A. AND DOWNEY, B.R. (1991). Presence of follicular fluid in the porcine oviduct and its contribution to the acrosome reaction. Mol. Reprod. Dev. 30, 148-153.

10. EISENBACH, M. AND TUR-KASPA, I. (1999). Do human eggs attract spermatozoa? BioEssays 21, 203210.

11. RALT, D., MANOR, M., COHEN-DAYAG, A., TURKASPA, I., MAKLER, A., YULI, I., DOR, J., BLUMBERG, S., MASHIACH, S. AND EISENBACH, M. (1994). Chemotaxis and chemokinesis of human spermatozoa to follicular factors. Biol. Reprod. 50, 774-785.

12. MAKLER, A., REICHLER, A., STOLLER, J. AND FEIGIN, P.D. (1992). A new model for investigating in real time the existence of chemotaxis in human spermatozoa. Fertil. Steril. 57, 1066-1074.

13. MAKLER, A., STOLLER, J., REICHLER, A., BLUMENFELD, Z. AND YOFFE, N. (1995). Inability of human sperm to change their orientation in response to external chemical stimuli. Fertil. Steril. 63, 10771082.

14. MANOR, M. (1994). Identification and purification of female-originated chemotactic factors. Ph.D. thesis, The Weizmann Institute of Science.

15. VILLANUEVA-DÍAZ, C., ARIAS-MARTÍNEZ, J., BERMEJO-MARTÍNEZ, L. AND VADILLOORTEGA, F. (1995). Progesterone induces human sperm chemotaxis. Fertil. Steril. 64, 1183-1188.

16. JAISWAL, B.S., TUR-KASPA, I., DOR, J., MASHIACH, S. AND EISENBACH, M. (1999). Human sperm chemotaxis: Is progesterone a chemoattractant? Biol. Reprod. 60, 1314-1319.

17. OLIVEIRA, R.G., TOMASI, L., ROVASIO, R.A. AND GIOJALAS, L.C. (1999). Increased velocity and induction of chemotactic response in mouse spermatozoa by follicular and oviductal fluids. J. Reprod. Fertil. 115, 23-27.

18. COHEN-DAYAG, A., RALT, D., TUR-KASPA, I., MANOR, M., MAKLER, A., DOR, J., MASHIACH, S.
AND EISENBACH, M. (1994). Sequential acquisition of chemotactic responsiveness by human spermatozoa. Biol. Reprod. 50, 786-790.

19. GIOJALAS, L.C. AND ROVASIO, R.A. (1998). Mouse spermatozoa modify their dynamic parameters and chemotactic response to factors from the oocyte microenvironment. Int. J. Androl. 21, 201-206.

20. EISENBACH, M. AND RALT, D. (1992). Precontact mammalian sperm-egg communication and role in fertilization. Am. J. Physiol. 262 (Cell Physiol. 31), C1095-C1101.

21. COHEN-DAYAG, A., TUR-KASPA, I., DOR, J., MASHIACH, S. AND EISENBACH, M. (1995). Sperm capacitation in humans is transient and correlates with chemotactic responsiveness to follicular factors. Proc. Natl. Acad. Sci. U.S.A. 92, 11039-11043.

22. JAISWAL, B.S., COHEN-DAYAG, A., TUR-KASPA, I. AND EISENBACH, M. (1998). Sperm capacitation is, after all, a prerequisite for both partial and complete acrosome reaction. FEBS Lett. 427, 309-313.

23. JAISWAL, B.S., EISENBACH, M. AND TURKASPA, I. (1999). Detection of partial and complete acrosome reaction in human spermatozoa: which inducers and probes to use? Mol. Hum. Reprod. 5, 214-219.

24. SUAREZ, S.S. (1998). The oviductal sperm reservoir in mammals: mechanisms of formation. Biol. Reprod. 58, 1105-1107.

25. OVERSTREET, J.W. AND DROBNIS, E.Z. (1991). Sperm transport in the female tract. In: Advances in donor insemination (C.L.R. Barratt and I.D. Cooke, eds.), pp. 33-49. Cambridge University Press, Cambridge, England.

26. SMITH, T.T. AND YANAGIMACHI, R. (1991). Attachment and release of spermatozoa from the caudal isthmus of the hamster oviduct. J. Reprod. Fertil. 91, 567-573.

27. LEFEBVRE, R. AND SUAREZ, S.S. (1996). Effect of capacitation on bull sperm binding to homologous oviductal epithelium. Biol. Reprod. 54, 575-582.

28. BRONSON, R. AND HAMADA, Y. (1977). Gamete interactions in vitro. Fertil. Steril. 28, 570-576.

29. BEDFORD, J.M. AND KIM, H.H. (1993). Cumulus oophorus as a sperm sequestering device, in vivo. J. Exp. Zool. 265, 321-328.

30. EISENBACH, M. (1995). Sperm changes enabling fertilization in mammals. Curr. Opin. Endocrinol. Diabetes 2, 468-475.

31. JAISWAL, B.S. AND EISENBACH, M. (1999). Capacitation. In: Fertilization (D.M. Hardy and D.L. Garbers, eds.), pp. In press. Academic Press, San Diego.

32. RALT, D., GOLDENBERG, M., FETTEROLF, P., THOMPSON, D., DOR, J., MASHIACH, S., GARBERS, D.L. AND EISENBACH, M. (1991). 
Sperm attraction of follicular factor(s) correlates with human egg fertilizability. Proc. Natl. Acad. Sci. U.S.A. $88,2840-2844$.

33. GNESSI, L., RUFF, M.R., FRAIOLI, F. AND PERT, C.B. (1985). Demonstration of receptor-mediated chemotaxis by human spermatozoa. A novel quantitative bioassay. Exp. Cell Res. 161, 219-230.

34. ANDERSON, R.A., FEATHERGILL, K.A., RAWLINS, R.G., MACK, S.R. AND ZANEVELD, L.J.D. (1995). Atrial natriuretic peptide: a chemoattractant of human spermatozoa by a guanylate cyclase-dependent pathway. Mol. Reprod. Dev. 40, 371378.

35. LEE, S.-L., KAO, C.-C. AND WEI, Y.-H. (1994). Antithrombin III enhances the motility and chemotaxis of boar sperm. Comp. Biochem. Physiol. 107A, 277-282.

36. IQBAL, M., SHIVAJI, S., VIJAYASARATHY, S. AND BALARAM, P. (1980). Synthetic peptides as chemoattractants for bull spermatozoa: structure activity correlations. Biochem. Biophys. Res. Commun. 96, 235-242.

37. ZAMIR, N., RIVEN-KREITMAN, R., MANOR, M., MAKLER, A., BLUMBERG, S., RALT, D. AND EISENBACH, M. (1993). Atrial natriuretic peptide attracts human spermatozoa in vitro. Biochem. Biophys. Res. Commun. 197, 116-122.

38. VILLANUEVA-DÍAZ, C., ARIAS-MARTÍNEZ, J., BUSTOS-LÓPEZ, H. AND VADILLO-ORTEGA, F. (1992). Novel model for study of human sperm chemotaxis. Fertil. Steril. 58, 392-395.

39. SLIWA, L. (1993). Effect of heparin on human spermatozoa migration in vitro. Arch. Androl. 30, 177181.

40. Sliwa, L. (1994). Chemotactic effect of hormones in mouse spermatozoa. Arch. Androl. 32, 83-88.

41. ZAMIR, N., SKOFITSCH, G., ESKAY, R. AND JACOBOWITZ, D.M. (1986). Distribution of immunoreactive atrial natriuretic peptide in the central nervous system of the rat. Brain Res. 365, 105-111.

42. VILLANUEVA-DÍAZ, C., VADILLO-ORTEGA, F., KABLY-AMBE, A., DIAZ-PEREZ, M.A. AND KRIVITZKY, S.K. (1990). Evidence that human follicular fluid contains a chemoattractant for spermatozoa. Fertil. Steril. 54, 1180-1182.

43. SLIWA, L. (1993). Heparin as a chemoattractant for mouse spermatozoa. Arch. Androl. 31, 149-152.

44. SLIWA, L. (1995). Chemotaction of mouse spermatozoa induced by certain hormones. Arch. Androl. 35, 105-110.

45. MILLER, R.L. (1982). Synthetic peptides are not chemoattractants for bull sperm. Gamete Res. 5, 395401.
46. EISENBACH, M. (1999). Mammalian sperm chemotaxis and its association with capacitation. Dev. Genet. 25, 87-94.

47. NAVARRO, M.C., VALENCIA, J., VAZQUEZ, C., COZAR, E. AND VILLANUEVA, C. (1998). Crude mare follicular fluid exerts chemotactic effects on stallion spermatozoa. Reprod. Domest. Anim. 33, 321-324.

\section{ABSTRACT
Does sperm chemotaxis have a role in fertilization?

\section{EISENBACH}

Sperm chemotaxis to follicular fluid has been established by a variety of means in human and mouse spermatozoa. It was found that only a small fraction of a given sperm population (averaging around $10 \%$ ) is chemotactically responsive and that this fraction constitutes capacitated (ripe) spermatozoa. Both the chemotactic responsiveness and the capacitated state are transient (with a lifetime between $50 \mathrm{~min}$ and $4 \mathrm{~h}$ ) and they occur only once in the sperm's lifetime. It has been proposed that the role of sperm chemotaxis in mammals (at least in man) is selective recruitment of capacitated spermatozoa for fertilizing the egg, and that the role of the continuous replacement of chemotactic/capacitated spermatozoa is to prolong the duration of time over which capacitated spermatozoa would be available in the female reproductive tract. The sperm chemoattractants have not been identified but they appear to be heatstable peptides. The in vivo location of sperm chemotaxis is not known; a number of possible locations are discussed.

Key words : chemotaxis, capacitation, chemokinesis, fertilisation, sperm motility, female reproductive tract. 